\title{
Age Dependent Progression of Multiple Epiphyseal Dysplasia and Pseudoachondroplasia Due to Heterozygous Mutations in COMP Gene
}

\author{
Nabil El-Lababidi', Marie Zikánová ${ }^{1}$, Alice Baxová ${ }^{1}$, Lenka Nosková1, \\ Alena Leiská ${ }^{3}$, Lukáš Lambert ${ }^{3}$, Tomáš Honzík ${ }^{1}$, Jiř́ Zeman ${ }^{1}$ \\ ${ }^{1}$ Department of Pediatrics and Inherited Metabolic Disorders, First Faculty \\ of Medicine, Charles University and General University Hospital in Prague, \\ Prague, Czech Republic; \\ ${ }^{2}$ Institute of Biology and Medical Genetics, First Faculty of Medicine, Charles \\ University and General University Hospital in Prague, Prague, Czech Republic; \\ ${ }^{3}$ Department of Radiology, First Faculty of Medicine, Charles University \\ and General University Hospital in Prague, Prague, Czech Republic
}

Received April 13, 2020; Accepted September 14, 2020.

Key words: Short stature - Multiple epiphyseal dysplasia - Pseudoachondroplasia COMP

\begin{abstract}
Dominantly inherited mutations in COMP gene encoding cartilage oligomeric matrix protein may cause two dwarfing skeletal dysplasias, milder multiple epiphyseal dysplasia (MED) and more severe pseudoachondroplasia (PSACH). We studied the phenotype and X-rays of 11 patients from 5 unrelated families with different COMP mutations. Whole exome and/or Sangers sequencing were used for molecular analyses. Four to ten X-ray images of hands hips, knees or spine were available for each patient for retrospective analyses. Eight patients with MED have mutation c.1220G >A and 3 children with PSACH mutations c.1359C >A, c.1336G >A, or the novel mutation c.1126G > T in COMP. Progressive failure in growth developed in all patients from early childhood and resulted in short stature $<3^{\text {rd }}$ percentile in 7 patients and very short stature $<1^{\text {st }}$ percentile in four. Most patients had joint pain since childhood, severe stiffness in shoulders and elbows but
\end{abstract}

This study was supported by projects Progres Q32, RVO VFN 64165 and PRIMUS/17/MED/6.

Mailing Address: Prof. Jiř́ Zeman, MD., PhD., Department of Pediatrics and Inherited Metabolic Disorders, First Faculty of Medicine, Charles University and General University Hospital in Prague, Ke Karlovu 2, 12100 Prague 2, Czech Republic; Mobile Phone: +420 606415 073; e-mail: jzem@lf1.cuni.cz 
increased mobility in wrists. Six children had bowlegs and two had knock knees. In all patients, X-rays of hands, hips and knees showed progressive, age-dependent skeletal involvement more pronounced in the epiphyses of long rather than short tubular bones. Anterior elongation and biconvex configuration of vertebral bodies were more conspicuous for kids. Six children had correction of knees and two adults had hip replacement. Skeletal and joint impairment in patients with MED and PSACH due to COMP mutation start in early childhood. Although the clinical severity is mutation and age dependent, many symptoms represent a continuous phenotypic spectrum between both diseases. Most patients may benefit from orthopaedic surgeries.

\section{Introduction}

Cartilage oligomeric matrix protein (COMP) encoded by COMP gene and synthesized by chondrocytes, is the multifunctional extracellular matrix calcium-binding glycoprotein involved in the enhancement of chondrocyte attachment, proliferation and cartilage production, maintenance, and homeostasis (Hecht et al., 2005; Merritt et al., 2006; Briggs et al., 2014; Posey et al., 2019). Mutations in COMP may cause two different types of severe short-limb dwarfism with autosomal dominant inheritance pattern affecting especially the epiphyses of long bones including multiple epiphyseal dysplasia (MED/EDM1, MIM132400) and pseudoachondroplasia (PSACH, MIM177170), but an overlap between both disorders was also described as a part of a continuous phenotypic spectrum (Jackson et al., 2012; Spranger et al., 2012).

In our study we analysed the clinical course of the disease and available $\mathrm{X}$-rays in 11 patients from 5 unrelated families with MED/EDM1 or PSACH due to different heterozygous mutations in COMP gene including c.1220G >A, c.1359C>A, c.1336G >A, and a novel mutation c.1126G > T.

\section{Material and Methods}

The study involved 11 patients from 5 unrelated families. All of them developed progressive failure in their growth from early childhood starting between the age of 2 and 7 years. This growth failure resulted in in short stature $<3^{\text {rd }}$ percentile in 7 patients and short stature $<1^{\text {st }}$ percentile in another four (Table 1). Most patients have stiff shoulders and elbows with severely restricted range of motion. Contrary, their wrists mobility is increased. Progressive and mostly painful gait disturbances due to legs deformities with bowlegs was present in 6 children and knock knees in two. Surgical correction of lower extremities was necessary in 6 patients during childhood and a hip replacement was required in the two oldest patients (Table 1). All patients have normal cognitive functions. Due to the lack of adequate mobility, four patients are overweight, and one is obese.

Genomic DNA from all of these patients and their parents was used for wholeexome sequencing (WES) in families A, C and E. Direct Sanger sequencing of COMP gene exons was performed in families B and D. Exome enrichment for WES was 


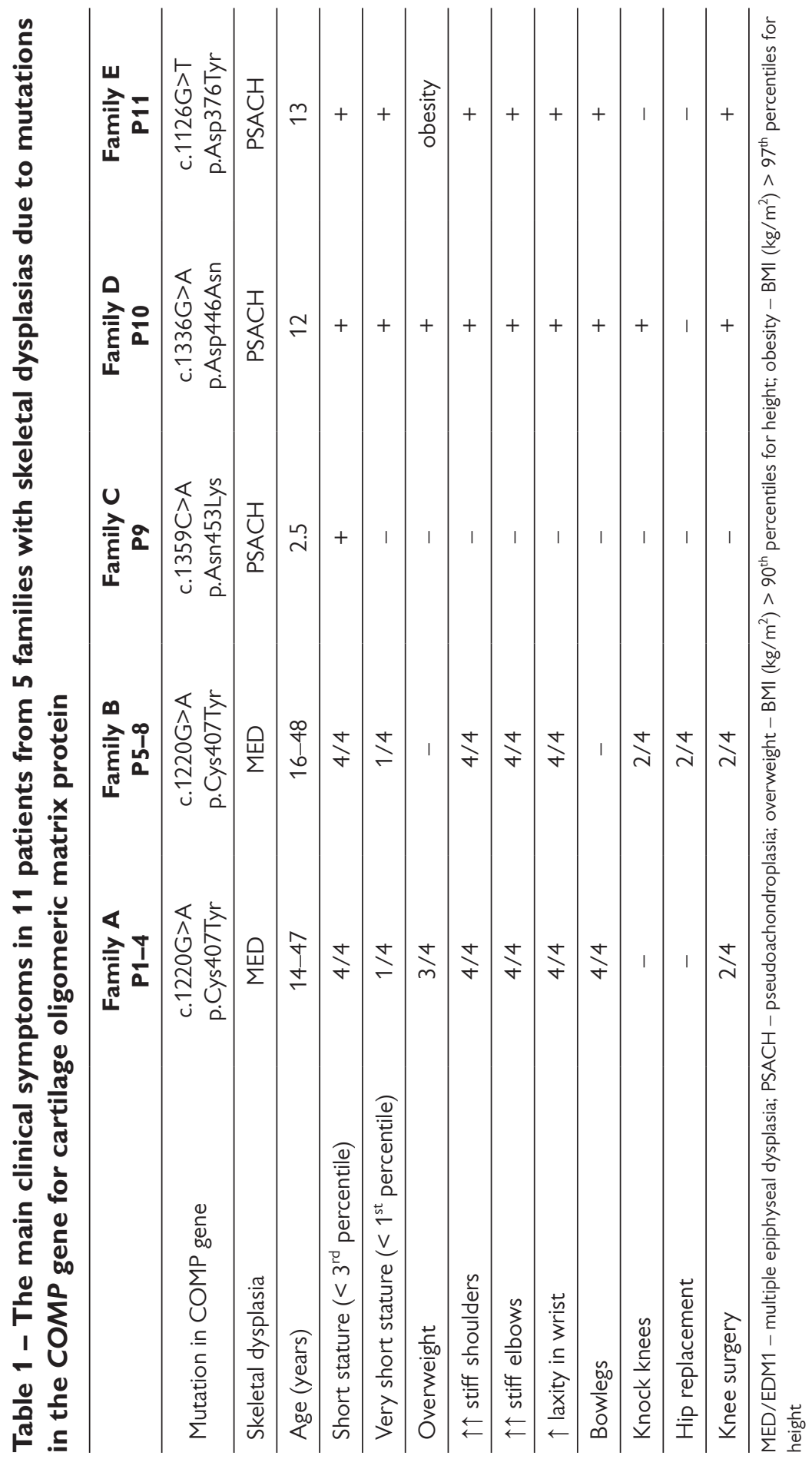


performed on individually barcoded samples using SeqCap EZ MedExome Probes (Roche, USA) and sequenced using HiSeq 2500 (Illumina, USA). Reads were aligned to the hg19 reference genome using NovoalignCS version 3.1.11.08 (Novocraft, Malaysia) with default parameters. Sequence variants in analysed samples were identified using the GATK SAMtools package (version 3.1.10.1.8). High confidence variants list (SNP qual > 100 and Indel qual > 50) was annotated using Annovar

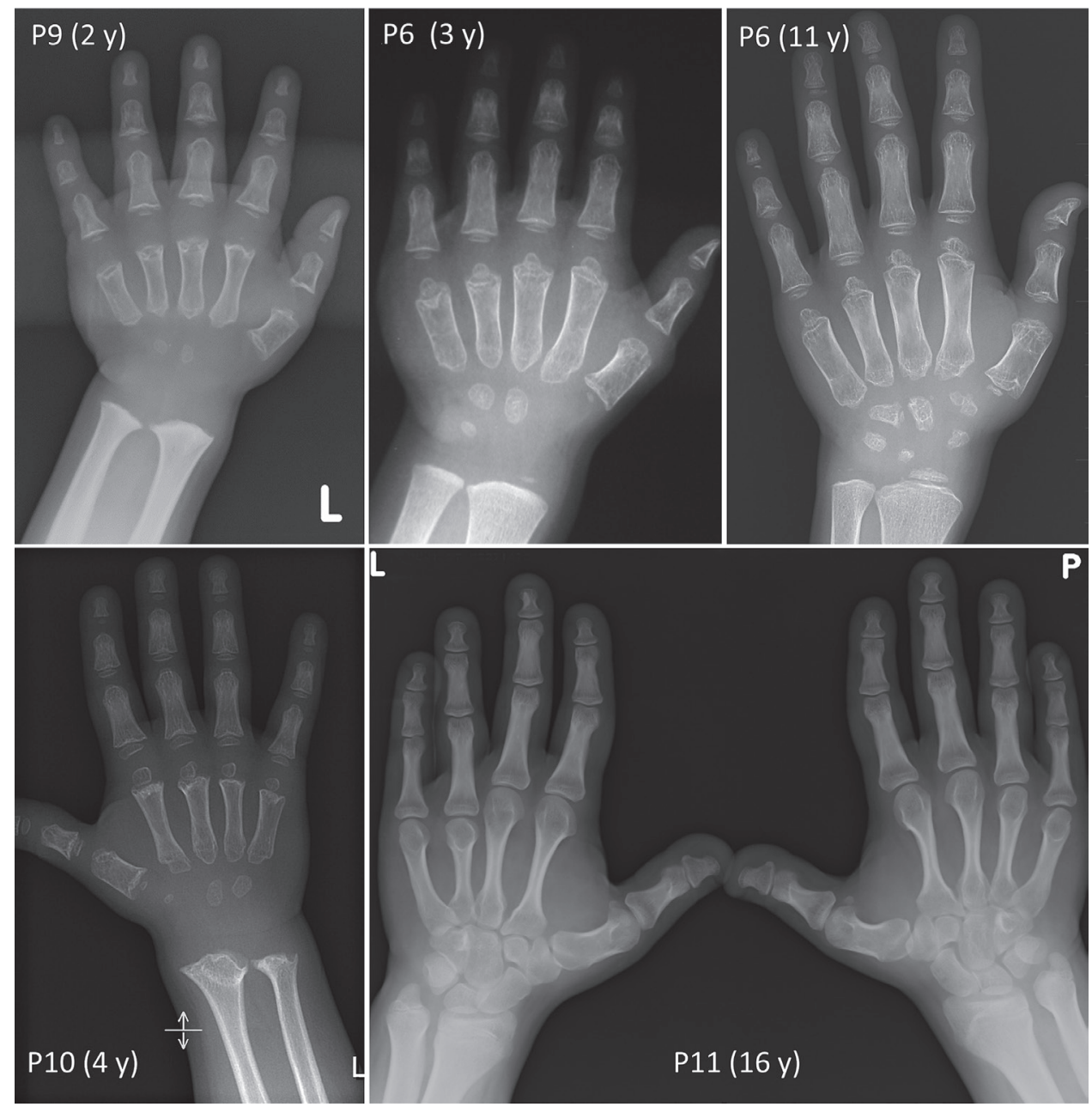

Figure 1 - X-ray survey of the hands and wrists since childhood to adulthood in patients P6, 9, 10 and 11 with multiple epiphyseal dysplasia or pseudoachondroplasia due to different mutations in COMP gene show deformity of the metaphyses of the shortened metacarpal bones with uneven surface. The shortening becomes more apparent after growth plate fusion. Mild shortening can be appreciated in the phalanges as well. The epiphyseal ossification centres of the metacarpal bones, distal radius and carpal bones are smaller and, in some patients, have irregular shape. The distal metaphyses of the radius and ulna have irregular contours and in adolescence show deformity that involves the radio-carpal and the distal radio-ulnar joint. The ossification is delayed. 
(hg19). Due to an assumed autosomal dominant mode of inheritance, variants present in affected individuals and not present in healthy relatives were taken into consideration. Genetic background of the diagnosis of MED was reviewed and genes associated with this disorder were considered first. Presence of candidate variants was confirmed by Sanger sequencing and segregation of the variant in the whole family was performed. Four to ten X-ray images of various bones and joins from different orthopaedic departments were available for each patient.

The study was approved by the Ethics Committee of the General University Hospital in Prague and was conducted in agreement with institutional guidelines. Written informed consent for molecular analyses was obtained from all patients or their parents.

\section{Results}

Molecular analyses in 8 patients with MED/EDM1 from families A and B revealed a dominantly inherited mutation c.1220G >A (p.Cys407Tyr) in COMP gene. De novo mutations c.1359C>A (p.Asn453Lys), c.1336G >A (p.Asp446Asn) and a new mutation c.1126G > T (p.Asp376Tyr) were found in another three patients from families $C$, D and $E$ (Table 1).

In all patients, $X$-rays of the hands, hips and knees showed age-dependent progression of epiphyseal changes, more pronounced in the epiphyses of long than short bones. Anterior elongation and biconvex configuration of vertebral bodies

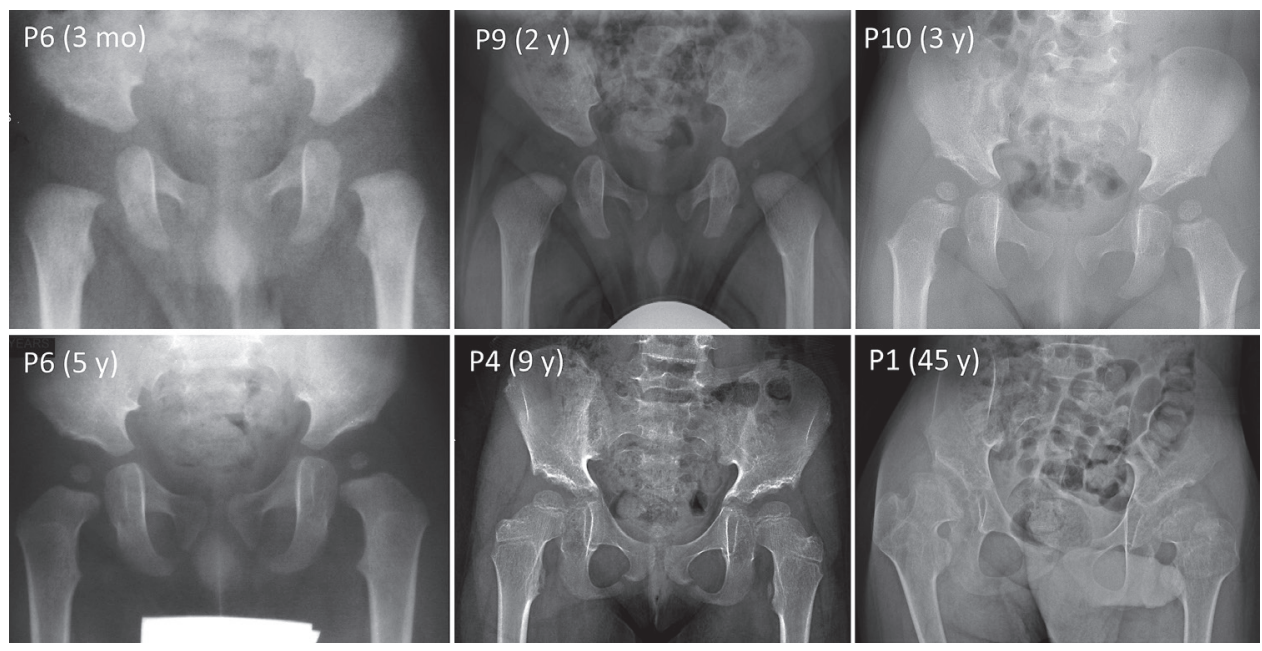

Figure 2 - X-ray survey of the hip joints since childhood to adulthood in patients P1, 4, 6, 9 and 10 with multiple epiphyseal dysplasia or pseudoachondroplasia due to different mutations in COMP gene show flattened, widened, robust femoral necks, decreased inclination of acetabular roofs that are shallow (dysplastic) with pointed lateral margins. The ossification centres of the femoral head are small. The iliac wings appear flared. Adult P1 developed early osteoarthritis in the setting of pre-existing severe deformity of the hip joint. 


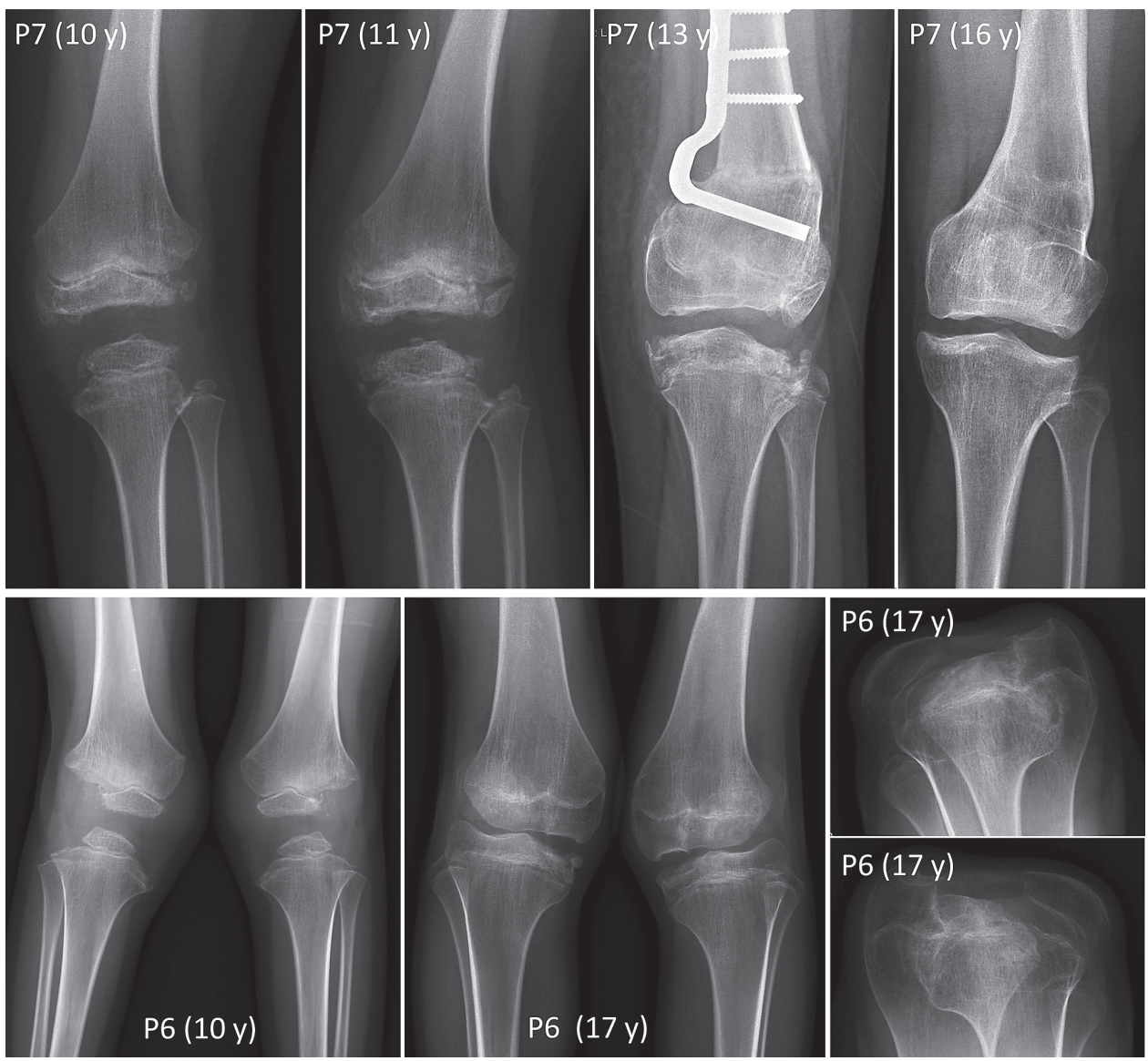

Figure 3 - X-ray survey of the knees (AP and axial views) of P6 and P7 with multiple epiphyseal dysplasia due to mutations C.1220G >A in COMP gene show progression of bone deformities during growth and ossification. The shortened metaphyses with irregular ossification progressed to deformity of their shape and axial deformity of the proximal tibia and distal femur (surgically corrected in P7), subluxation both in the femoro-tibial and femoro-patellar part of the joint in P7.

were more conspicuous in younger children. The selection of $\mathbf{X}$-ray images of the wrists, pelvis, knees and spine in different age groups is shown in Figures 1-4.

\section{Discussion}

More than 300 mutations in COMP gene have already been identified in patients with two different dwarfing conditions including multiple epiphyseal dysplasia (MED/EDM1) and pseudoachondroplasia (PSACH) (Briggs et al., 2014; Chen et al., 2019). Although both disorders still represent individual diseases, an overlap between them was suggested on clinical and radiologic levels (Spranger et al., 2012). The first symptoms usually start in toddlers or preschool children with progression 


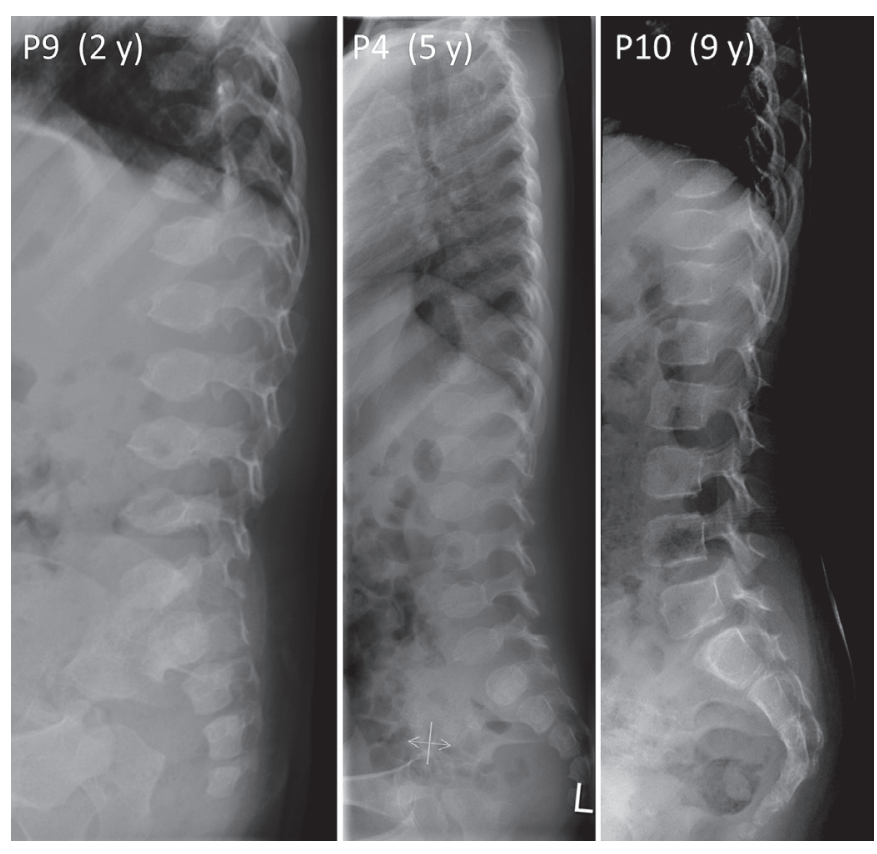

Figure 4 - X-ray survey of the lateral views of the spine of P4, 9 and P10 with multiple epiphyseal dysplasia or pseudoachondroplasia due to different mutations in COMP gene show anterior elongation and biconvex configuration of vertebral bodies that are more conspicuous in two younger probands.

of leg deformities and gait and growth disturbances resulting in a very short stature. Although X-ray images may help with the diagnosis of skeletal involvement, a molecular testing is usually necessary for definitive diagnostics (Anthony et al., 2015). Most patients with PSACH have a mutation in COMP gene. The most common is mutation Asp469del, which impedes trafficking of COMP and type IX collagen in chondrocytes (Chen et al., 2008). Contrary to PSACH, multiple epiphyseal dysplasia may be except mutation in COMP gene also caused by mutations in other genes including COL9A1 for collagen type IX $\alpha-1$, COL9A2 for collagen type IX $\alpha-2$, COL9A3 for collagen type IX $\alpha-3$, and MATN3 for matrilin-3 resulting in similarly disorganized epiphyseal ossification and destruction of the articular cartilage (Jackson et al., 2004; Hecht et al., 2005; Spranger et al., 2012). In addition, the autosomal recessive variant of MED is caused by a mutation of the sulphate transporter gene SLC26A2 (Anthony et al., 2015). Therefore, MED belongs to the most genetically heterogeneous disorders between skeletal dysplasias (Unger and Hecht, 2001). Mutations in specific residues or regions of COMP are significantly associated with either PSACH or MED but other factors including genetic modifiers are likely to influence the disease severity, which has already been reported in MED caused by MATN3 mutations (Briggs et al., 2014).

In our patients with heterozygous mutations in COMP, the most common variant was c. $1220 \mathrm{G}>\mathrm{A}$ identified in 8 patients with MED from two unrelated families. The variant is present in public Human Gene Mutation Database and according 
to the American College of Medical Genetics and Genomics classification is likely pathogenic. It is located in a well-established functional domain (calcium binding type III repeats), where no benign variations are present. The mutation c.1220G>A was previously reported in a sporadic Chinese girl with PSACH (Cao et al., 2011). Her radiographs showed platyspondyly of the spine and anterior fractures of the vertebrae, significant epiphyseal and metaphyseal changes in the joints of the long and short tubular bones, short metacarpal bones and phalanges and small irregular carpal bones compatible with PSACH (Cao et al., 2011). Unlike the Chinese girl, our patients with the same mutation lack a severe impairment of the spine and their phenotypes are more compatible with MED. Our results suggest that not only different mutations, but also the same variant in COMP gene may cause either multiple epiphyseal dysplasia or pseudoachondoplasia. This is similar to other amino acid changes in the same protein position as p.Cys407Tyr resulting in MED/EDM1 (Kennedy et al., 2005) or PSACH (Chen et al., 2019). This is supported by experiments described in a CollITg ${ }^{\text {cog }}$ mouse.

The induction of endoplasmic reticulum (ER) stress in the proliferative zone of chondrocytes produced decreased cell proliferation and bone growth and demonstrated the central role of classical ER stress in the MED and PSACH disease process. However, the induced ER stress was not sufficient enough to replicate all of the pathological features of MED and PSACH, suggesting that a combination of more factors including the presence of a defective cartilage extracellular matrix in addition to an increased ER stress is likely to contribute to the pathogenesis of MED and PSACH (Kung et al., 2015; Posey et al., 2019).

Mutations p.Asp376Tyr, p.Asn453Lys and p.Asp446Asn are also located within the type III repeat domain of COMP, which is the most common domain for both MED and PSACH. Mutation p.Asp376Tyr was published previously in a patient with PSACH, who accumulated COMP and type IX collagen in chondrocytes (Maddox et al., 1997). Mutation p.Asp446Asn has not been described yet, but 3 different substitutions of Asp446 were published in two patients with MED (Zankl et al., 2007; Kim et al., 2011) and one patient with PSACH (Kennedy et al., 2005).

The quality of life is strongly affected in patients with a mutation in COMP gene. Treatment is based on the severity of limb deformity, the level of joint destruction, age of the patient and his/her needs. In our group of patients, the most common orthopaedic surgeries during childhood were focused on bowlegs and knock knees. Especially children can benefit greatly from limb realignment procedures. Adults may also have good functional outcomes with joint arthroplasty (Anthony et al., 2015), but discussing the proper timing for the surgery may be of importance. Interestingly, adolescents with idiopathic scoliosis (AIS) have higher COMP promoter methylation and lower gene expression, which correlates with young age and high Cobb angle of the main curve. COMP promoter methylation may provide prognostic information in predicting the susceptibility and curve progression of AIS (Mao et al., 2018). 


\section{Conclusion}

In both multiple epiphyseal dysplasia and pseudoachondroplasia, the skeletal and joint impairment due to mutation in COMP start in early childhood. Although the clinical severity is mutation and age dependent, many symptoms represent a continuous phenotypic spectrum between both diseases. Most patients may benefit from orthopaedic surgeries, but the disease still has severe negative impact on their quality of life. Early establishing of the diagnosis is important for genetic counselling and eventual pre-implantation genetic testing.

\section{References}

Anthony, S., Munk, R., Skakun, W., Masini, M. (2015) Multiple epiphyseal dysplasia. J. Am. Acad. Ortop. Surg. 23(3), 164-172.

Briggs, M. D., Brock, J., Ramsden, S. C., Bell, P. A. (2014) Genotype to phenotype correlations in cartilage oligomeric matrix protein associated chondrodysplasias. Eur. J. Hum. Genet. 22(11), 1278-1282.

Cao, L. H., Wang, L. B., Wang, S. S., Ma, H. W., Ji, C. Y., Luo, Y. (2011) Identification of novel and recurrent mutations in the calcium binding type III repeats of cartilage oligomeric matrix protein in patients with pseudoachondroplasia. Genet. Mol. Res. 10(2), 955-963.

Chen, J., Zhang, W., He, J., Zhang, R., Cao, Y., Liu, X. (2019) A novel mutation in exon 11 of COMP gene in a Chinese family with pseudoachondroplasia. Genes Dis. 6(1), 47-55.

Chen, T. L., Posey, K. L., Hecht, J. T., Vertel, B. M. (2008) COMP mutations: Domain-dependent relationship between abnormal chondrocyte trafficking and clinical PSACH and MED phenotypes. J. Cell. Biochem. 103(3), 778-787.

Hecht, J. T., Hayes, E., Haynes, R., Cole, W. G. (2005) COMP mutations, chondrocyte function and cartilage matrix. Matrix Biol. 23(8), 525-33.

Jackson, G. C., Barker, F. S., Jakkula, E., Czarny-Ratajczak, M., Mäkitie, O., Cole, W. G., Wright, M. J., Smithson, S. F., Suri, M., Rogala, P., Mortier, G. R., Baldock, C., Wallace, A., Elles, R., Ala-Kokko, L., Briggis, M. D. (2004) Missense mutations in the beta strands of the single A-domain of matrilin-3 result in multiple epiphyseal dysplasia. J. Med. Genet. 41(1), 52-59.

Jackson, G. C., Mittaz-Crettol, L., Taylor, J. A., Mortier, G. R., Spranger, J., Zabel, B., Le Merrer, M., Cormier-Daire, V., Hall, C. M., Offiah, A., Wright, M. J., Savarirayan, R., Nishimura, G., Ramsden, S. C. Elles, R., Bonafe, L., Superti-Furga, A., Unger, S., Zankl, A., Briggs, M. D. (2012) Pseudoachondroplasia and multiple epiphyseal dysplasia: A 7-year comprehensive analysis of the known disease genes identify novel and recurrent mutations and provides an accurate assessment of their relative contribution.

Hum. Mutat. 33(1), 144-157.

Kennedy, J., Jackson, G., Ramsden, S., Taylor, J., Newman, W., Wright, M. J., Donnai, D., Elles, R., Briggs, M. D. (2005) COMP mutation screening as an aid for the clinical diagnosis and counselling of patients with a suspected diagnosis of pseudoachondroplasia or multiple epiphyseal dysplasia. Eur. J. Hum. Genet. 13(5), 547-555.

Kim, O. H., Park, H., Seong, M. W., Cho, T. J., Nishimura, G., Superti-Furga, A., Unger, S., Ikegawa, S., Choi, I. H., Song, H. R., Kim, H. W., Yoo, W. J., Shim, J. S., Chung, C. Y., Oh, C. W., Jeong, C., Song, K. S., Seo, S. G., Cho, S. I., Yeo, I. K., Kim, S. Y., Park, S., Park, S. S. (2011) Revisit of multiple epiphyseal dysplasia: Ethnic difference in genotypes and comparison of radiographic features linked to the COMP and MATN3 genes. Am. J. Med. Genet. A 155A(11), 2669-2680. 
Kung, L. H., Rajpar, M. H., Preziosi, R., Briggs, M. D., Boot-Handford, R. P. (2015) Increased classical endoplasmic reticulum stress is sufficient to reduce chondrocyte proliferation rate in the growth plate and decrease bone growth. PLoS One 10(2), e0117016.

Maddox, B. K., Keene, D. R., Sakai, L. Y., Charbonneau, N. L., Morris, N. P., Ridgway, C. C., Boswell, B. A., Sussman, M. D., Horton, W. A., Bächinger, H. P., Hecht, J. T. (1997) The fate of cartilage oligomeric matrix protein is determined by the cell type in the case of a novel mutation in pseudoachondroplasia. J. Biol. Chem. 272(49), 30993-30997.

Mao, S. H., Qian, B. P., Shi, B., Zhu, Z. Z., Qiu, Y. (2018) Quantitative evaluation of the relationship between COMP promoter methylation and the susceptibility and curve progression of adolescent idiopathic scoliosis. Eur. Spine J. 27(2), 272-277.

Merritt, T. M., Alcorn, J. L., Haynes, R., Hecht, J. T. (2006) Expression of mutant cartilage oligomeric matrix protein in human chondrocytes induces the pseudoachondroplasia phenotype. J. Orthop. Res. 24(4), 700-707.

Posey, K. L., Coustry, F., Veerisetty, A. C., Hossain, M. G., Gambello, M. J., Hecht, J. T. (2019) Novel mTORC1 mechanism suggests therapeutic targets for COMPopathies. Am. J. Pathol. 189(1), 132-146.

Spranger, J. W., Brill, P. W., Superti-Furga, A., Unger, S., Nishimura, G. (2012) Bone Dysplasias: An Atlas of Genetic Disorders of Skeletal Development, $3^{\text {rd }}$ Edition. Oxford University Press, Oxford.

Unger, S., Hecht, J. T. (2001) Pseudoachondroplasia and multiple epiphyseal dysplasia: New etiologic developments. Am. J. Med. Genet. 106(4), 244-250.

Zankl, A., Jackson, G. C., Crettol, L. M., Taylor, J., Elles, R., Mortier, G. R., Spranger, J., Zabel, B., Unger, S., Merrer, M. L., Cormier-Daire, V., Hall, C. M., Wright, M. J., Bonafe, L., Superti-Furga, A., Briggs, M. D. (2007) Preselection of cases through expert clinical and radiological review significantly increases mutation detection rate in multiple epiphyseal dysplasia. Eur. J. Hum. Genet. 15(2), 150-154.

El-Lababidi N.; Zikánová M.; Baxová A.; Nosková L.; Leiská A.; Lambert L.; Honzík T.; Zeman J. 